Document downloaded from:

http://hdl.handle.net/10251/64109

This paper must be cited as:

Herrero Durá, JM.; Blasco, X.; Sánchez Pérez, JV.; Redondo, J. (2016). Design of sound phase diffusers by means of multiobjective optimization approach using ev-MOGA evolutionary algorithm. Structural and Multidisciplinary Optimization. 53(4):861-879. doi:10.1007/s00158-015-1367-0.

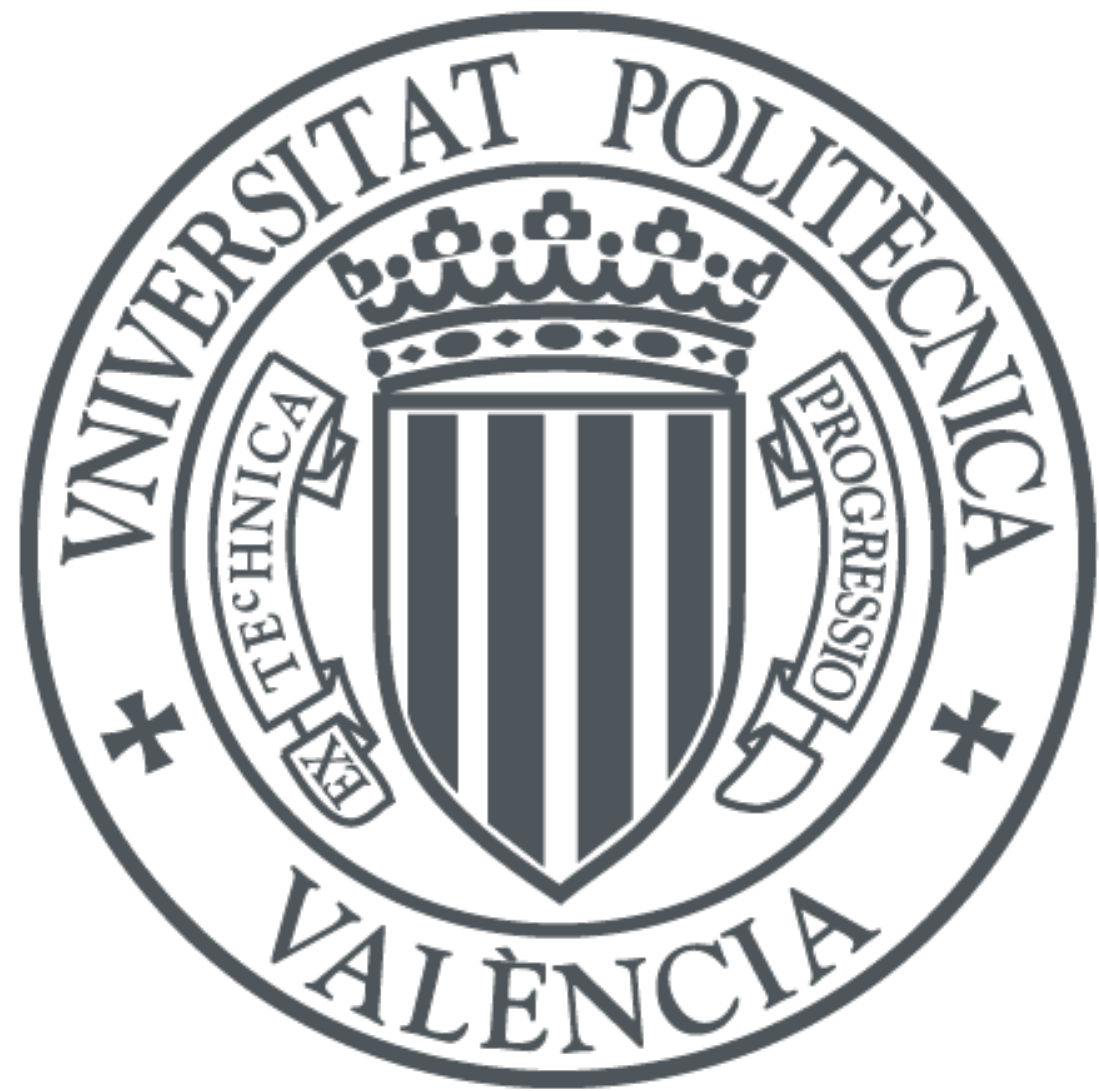

The final publication is available at

http://dx.doi.org/10.1007/s00158-015-1367-0

Copyright Springer Verlag

Additional Information 


\title{
Design of sound phase diffusers by means of multiobjective optimization approach using ev-MOGA evolutionary algorithm
}

\author{
J.M. Herrero · X. Blasco · J.V. Sánchez-Pérez · J. Redondo
}

Received: date / Accepted: date

\begin{abstract}
In this paper a new approach to design sound phase diffusers is presented. The acoustic properties of such diffusers are usually increased by using single objective optimization methods. Here we propose the use of a multiobjective (MO) approach to design them in order to take into account several conflicting characteristic simultaneously. Three different MO problems are posed to consider various scenarios where fundamentally the objective is to maximize the normalized diffusion coefficient (following the corresponding Audio Engineering Society standard) for the so-called medium frequencies. This single objective could be divided into other several objectives to adjust performances to designer preferences. A multi-objective evolutionary algorithm (called ev-MOGA) is used to characterize the Pareto front in a smart way. ev-MOGA is modified, by using integer codification and tuning some of its genetic operators, to adapt it to the new requirements. Special interest is posed in selecting the diffusers codification properly to eliminate duplicities that would produce a multimodal problem. Precision in the manufacturing process is taking into account in the diffuser codification causing, that the number of different diffusers are quan-
\end{abstract}

Partially supported by EVO-CONTROL project (ref. PROMETEO/2012/028, Generalitat Valenciana - Spain) and MEC (Spanish goverment) under grant $\mathrm{N}^{\circ}$ MTM2012-36740-C0202 .

J.M. Herrero $^{1}$ · X. Blasco ${ }^{1}$, J.V. Sánchez-Pérez ${ }^{2}$.

J. Redondo ${ }^{3}$

(1) Instituto Universitario de Automática e Informática industrial, ai2. (2) Centro de Tecnologías Físicas: Acústica, Materiales y Astrofísica. (3) Instituto para la Gestión Integrada de las zonas Costeras.

Universitat Politècnica de València, Camino de vera s/n, 46022 Valencia, Spain.

E-mail: juaherdu@isa.upv.es tified. Robust considerations related with the precision manufacturing process are considered in the decision making process. Finally, an optimal diffuser is selected considering designer preferences.

Keywords Sound diffusers · 1-D Schroeder diffuser ·

Multiobjective optimization · Evolutionary algorithms

\section{Introduction}

Sound diffusers can be defined as surfaces on which the sound is reflected in a non-specular way, that is, Snell' s law is not satisfied. These devices are used to improve the acoustic performance of rooms, spreading uniformly sound waves in multiple directions and therefore increasing the envelopment of the sound field and reducing echoes and focalizations. Schroeder first proposed these acoustic devices. The so-called Schroeder or Phase diffusers are formed by surfaces with series of reflecting wells with the same width and different depths separated by thin fins. If the variation of the depth of the wells is only in one direction, the resultant diffusers are called $1 D$ Schroeder diffusers. Fig. 1.a shows a picture of this kind of devices.

It is possible to improve largely the acoustic performance of such devices using several optimization techniques, but in all cases, the optimization processes in the field of sound diffusers have been carried out usually with a single objective function. In 1995 Cox [3] suggested the use of iterative methods as downhill simplex and quasi-Newton methods as tools to optimize these diffusers. In this case, the objective function used was the " diffusion parameter" , defined as the standard error of the sound pressure over the measurement positions, averaged over the desired working frequency range. In 
order to avoid low performance for particular frequencies within that range, a penalty is introduced adding the standard error to the frequency averaging. In recent years, volumetric diffusers based on arrays of cylindrical scatterers have been developed using evolutionary algorithms along to a two-dimensional Fourier approximation [8]. In this case, the algorithms are used to minimize the standard diffusion coefficient, and the optimization is carried out by removing or varying the diameter of a set of cylinders located in a periodic rectangular array, creating pseudorandom arrays with a high diffusion performance.

The aim of this work is to investigate the potential of an optimization comprehensive method for designing diffusers based on the Schroeder ones, applied to a 1D Schroeder diffusers formed by seven wells. However, unlike optimization processes used so far in which a single objective function is considered, we propose here the use of a multiobjective (MO) approach [14] that allows the obtaining of devices taking into account several objectives simultaneously. For instance, optimizing the diffusion coefficient for different ranges of frequencies separately.

To do that, we will use a Generate-First Choose c) Later (GFCL) approach ${ }^{1}$ [12]. In this approach we first obtain the Pareto set and its associated front. Each point of this Pareto set represents one solution to the $\mathrm{MO}$ problem that is neither better nor worse than any of the other points in the set. These are non-dominated points. Obtaining the Pareto front can help the designer to know the trade-off between the objectives and to choose the final diffuser.

To solve MO problem we propose to use Evolutionary Algorithms (MOEAs), which allow several solutions of the Pareto front will be generated simultaneously thanks to the populational nature of EAs $[1,21]$. MOEAs have capacity to handle a wide variety of problems with different degrees of complexity, permitting to characterize disjoint and non-convex Pareto fronts and having greater ease to avoid local minima.

In this work, the ev-MOGA algorithm ${ }^{2}[5,7]$ has been softly modified to be applied in the multiobjective optimization of the proposed 1-D diffuser. The essential properties of ev-MOGA are kept although an integer codification of the search space (that avoids different but equivalent diffusers, that means avoiding multimodality) and new genetic operators are used to adapt

\footnotetext{
${ }^{1}$ We do not use an aggregate objective function, to merge the different objectives. So we will avoid to weight the different objectives a priori.

2 Available for Matlab $\S$ at http://www.mathworks.com/ matlabcentral/fileexchange/31080-ev-moga-multiobjectiveevolutionary-algorithm.
}

ev-MOGA to the particular problem. ev-MOGA characterizes the Pareto front in a smart way producing higher density of solutions in areas where the Pareto front has a major trade-off. This smart Pareto front helps the designer to analyze it and to choose the final solution since it is a small and effective representation of the Pareto front trade-off [11].

The article is organized as follows: section 2 presents the mathematical foundations about the theory of the diffusers and the multiobjective approach followed; section 3 shows the ev-MOGA algorithm with its new codification and genetic operators; in section 4 three different $\mathrm{MO}$ problems are set to take into account different scenarios. Robustness of the Pareto solutions are analyzed and a diffuser is selected as a final solution; finally some concluding remarks are provided in section 5 .

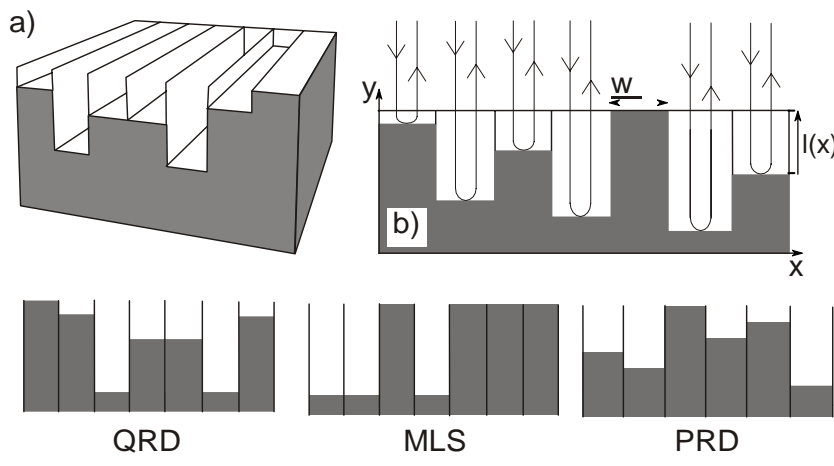

Fig. 1 (a) A scheme of 1-D Schroeder diffuser; (b) Explanation of the acoustic response of these diffusers; (c) Sectional views of QR, MLS and PR diffusers. $I(x)$ represents the depth profile of the diffuser. $w$ is the width of each well.

\section{Theoretical considerations}

\subsection{Theory of the Schroeder diffusers}

The underlying physics involved in the acoustic response of Schroeder diffusers can be explained as follows. The incident plane waves generated in the room penetrate into the wells, which have different depths, and afterwards are reflected from the bottom of the wells to the external space of the room, traveling acoustic paths of different length. The acoustic pressure at any point of the room is given by the interference between all the reflected waves generated at each well. If we assume that there are no energy losses, all the reflected waves will have the same amplitude but varied phases, in such a way the distribution of the well depths determine the polar distribution of the reflected pressure obtained by 
the whole diffuser [4]. A schematic illustration of this physical process can be seen in Fig. 1.b.

On the other hand, the far-field scattered pressure by a corrugated surface can be obtained by means of the Fraunhofer (or Fourier) theory. In the case of a 1D structure the reflected sound can be expressed using this theory, as [4]:

$$
p(a)=A_{0}{ }_{-X_{L}}^{s} e^{-2 \pi j\{\{2 /(x)] / \lambda\}} e^{2 \pi j\{[\sin \alpha] / \lambda\} x} d x
$$

where $l(x)$ is the local depth, $x$ is the coordinate along the surface, $\boldsymbol{a}$ is the scattering angle, $\lambda$ is the wavelength, and $A_{0}$ is a constant determined by the amplitude of the incident wave. Eq. (1) is applicable to sound waves under normal incidence, and it is assumed that the surface admittance varies in the $\mathrm{x}$ direction. The limits of the integral expression are defined by the boundaries of the sound diffuser, being $2 X_{L}$ the total width of the diffuser. This equation enables estimations of the far field diffusion as the Fourier Transform of the spatial depth profile of the diffuser surface $l(x)$. In other words, if the depth profile of the diffuser surface has a flat Fourier transform, sound will be reflected uniformly in all possible directions. So, the depth profile has to be designed following a numerical sequence with a flat Fourier Transform. Several numerical sequences have been used since the invention of sound diffusers. The most extended are Maximum Length Sequences (MLS) [17], Quadratic Residues (QR) [18] and Primitive Root Sequences (PRS) [19]. Fig. 1.c illustrates an example of each one of the named sequences.

There is a range of methods to predict the sound pressure reflected from a diffuser, from the simplest but faster to the numerically exact but computationally slow. Fraunhofer theory, introduced above, is the simplest way to predict the sound pressure reflected by a surface, but as a counterpart is the less accurate. At the other extreme of prediction methods, Cox demonstrated that the more accurate method to predict the scattering from diffusers is the Boundary Element Methods (BEM) [2] by comparison with experimental data. More recently it has been shown that Finite Element Methods (FEM) and Finite Difference Time Domain (FDTD) has the same ability to predict the sound reflected by rough surfaces [15]. The last method has the additional advantage that allows evaluating the temporal dispersion that diffusers produce.

However, due to the excessive computational cost involved in the use of these numerical methods (BEMFEM-FDTD), we have decided to use a less accurate analytical model but with a much lower computational cost, the Fraunhofer theory. It provides us reasonable values of the diffused field corresponding to each one of the individuals considered in the optimization process and, more importantly, give us the possibility to present the multiobjective optimization comprehensive method for designing Phase diffusers, which is the main goal of this paper.

\subsection{Quantification of the performance of sound diffusers}

In order to quantify the performance we have followed the ISO (International Organization for Standardization) standard [9]. It is based on the measurement of the acoustic pressure of the reflected sound over a range of angles, between $-90^{\circ}$ to $90^{\circ}$ in steps of $5^{\circ}$ (37 measurements in total). For this purpose a microphone is moved along a semi-circumference centered in the middle point of the test sample, which is composed by an array of four diffusers (with seven wells each one), see Fig. 2. Appropriate windowing of the signal allows the elimination of the direct sound. The parameter measured using this technique is known as the diffusion coefficient:

$$
d_{j}^{*}=\frac{-n_{i=1}^{n} p_{i j}^{2-2}-{ }_{i=1}^{n} p_{i j}^{2} \cdot 2}{(n-1)-n}
$$

where $d_{j}^{*}$ is the diffusion coefficient for the $\mathrm{j}$-th onethird octave band considered, $p_{i j}$ is the sound pressure of the reflected sound defined in Eq. (1) for the j-th onethird octave band considered at the $\mathrm{i}$-th measurement position, and $n$ is the number of measurement positions $(n=37)$. The value of the diffusion coefficient for each one-third octave band has been calculated considering the average of three logarithmically spaced frequencies within the band. We have checked that with this averaging, we obtain reliable results and, at the same time, the calculations are carried out with a low computational cost.

To normalize this diffusion coefficient from zero to one, $d_{j}^{*}$ is compared with a flat surface. The purpose of normalization is to remove edge diffraction scattering effects due to the limit size of the sample under analysis. In doing so, normalized diffusion coefficient represents the uniformity of scattering from the surface topology only. Concave surfaces should cause negative values for the normalized diffusion coefficient, but typically, it will be in the range from zero to one. Thus, the normalized diffusion coefficient, $d_{j}$, for the $j$-th one-third octave band considered, is defined as: 
$d_{j}=\frac{d^{j}-d_{j, \text { ref }}}{1-d_{j, \text { ref }}}$

$0 \leq d_{j} \leq 1$

where $d_{j}$,ref is the diffusion coefficient of a flat panel for the $j$-th one-third octave band considered. $d_{j}$ is equal to zero for flat surfaces and for all frequencies.

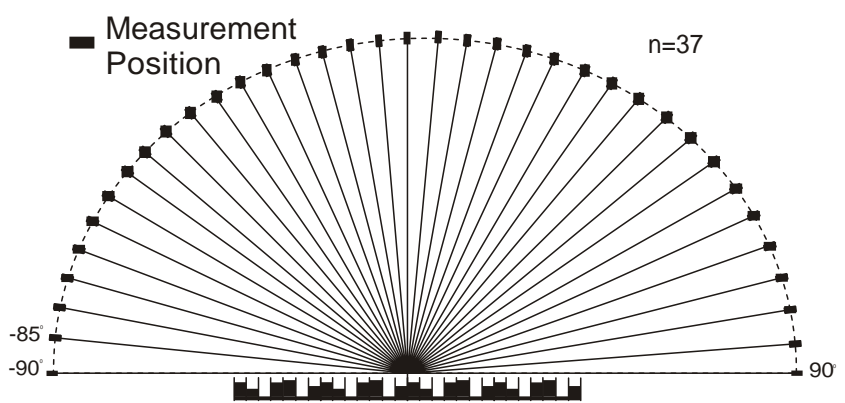

Fig. 2 Schematic measurement set up to obtain the diffusion coefficient of a sample composed by an array of four diffuser. Each diffuser has a width of $0.9 \mathrm{~m}$. Then $X_{L}$ in Eq. (1) is $1.8 \mathrm{~m}$. The wells width is $w=0.9 / 7=0.128 \mathrm{~m}$.

The bandwidth of a Schroeder diffuser is limited at high frequencies by the well width and at low frequencies by the maximum depth. In the present paper we are interested in the so-called medium frequency range which includes six one-third octave bands $\left(f_{1}=\right.$ $400 \mathrm{~Hz}, f_{2}=500 \mathrm{~Hz}, f_{3}=630 \mathrm{~Hz}, f_{4}=800 \mathrm{~Hz}, f_{5}=$ $1000 \mathrm{~Hz}$ and $f_{6}=1250 \mathrm{~Hz}$ ). To achieve significant diffusion in this frequency range we have chosen the following dimensions: the maximum depth will be limited to $0.320 \mathrm{~m}$, that for a standard QR diffuser supposes a minimum frequency (design frequency) falling at the beginning of the medium frequency range selected [4]. On the other hand, we have considered a total width of $0.9 \mathrm{~m}$, causing the maximum frequency to fall in the upper part of the considered frequency range [4]. Note that the Fraunhofer theory is valid only when the pressure field in each well of the diffuser do not create transverse modes, in other words, when the well width is smaller than half the minimum wavelength. In our case, the upper frequency correctly simulated is $1332 \mathrm{~Hz}$, which is over the central frequency of the highest one-third octave band considered in this work $(1250 \mathrm{~Hz})$. Then, under these conditions, we want to obtain the depth profile of the wells that maximizes $d_{j}$ in the medium frequency range $\left(j \in[1,2, \ldots, 6]\right.$ corresponding to $\left[f_{1}, f_{2}, \ldots, f_{6}\right]$ frequencies above mentioned).

\subsection{Multiobjective problem approach}

The MO problem can be formulated, in a general way, as follows ${ }^{3}$ :

$\min \boldsymbol{J}(\boldsymbol{\theta})=\min \left[J_{1}(\boldsymbol{\theta}), J_{2}(\boldsymbol{\theta}), \ldots, J_{s}(\boldsymbol{\theta})\right]$

subject to:

$\theta_{l i} \leq \theta_{i} \leq \theta_{u i},(1 \leq i \leq L)$

where $J_{i}(\boldsymbol{\theta}), i \in B:=[1 \ldots s]$ are the objectives to be minimized, $\boldsymbol{\theta}$ is a solution inside the $L$-dimensional solution space $D \subseteq R^{L}$, and $\theta_{\boldsymbol{l} i}$ and $\theta_{u i}$ are the lower and upper constraints that define the solution searching space $D$.

To solve the MO problem, we have to obtain the Pareto optimal set $\boldsymbol{\Theta}_{P}\left(\boldsymbol{J}\left(\boldsymbol{\Theta}_{P}\right)\right.$ denotes the Pareto front $)$, which contains the solutions where none dominates any of the others. Pareto dominance is defined as follows:

A solution $\boldsymbol{\theta}^{\mathbf{1}}$ dominates another solution $\boldsymbol{\theta}^{2}$, denoted by $\boldsymbol{\theta}^{1}<\boldsymbol{\theta}^{2}$, if

$$
\forall i \in B, J_{i}\left(\boldsymbol{\theta}^{1}\right) \leq J_{i}\left(\boldsymbol{\theta}^{2}\right) \wedge \exists k \in B: J_{k}\left(\boldsymbol{\theta}^{1}\right)<J_{k}\left(\boldsymbol{\theta}^{2}\right) .
$$

Therefore, the Pareto optimal set of solutions $\boldsymbol{\Theta}_{P}$ is given by

$\boldsymbol{\Theta}_{P}=\{\boldsymbol{\theta} \in D \mid \$ \tilde{\boldsymbol{\theta}} \in D: \tilde{\boldsymbol{\theta}}<\boldsymbol{\theta}\}$.

$\boldsymbol{\Theta}_{P}$ is unique and normally includes infinite solutions. Hence a set $\boldsymbol{\Theta}_{p}$, with a finite number of elements from $\boldsymbol{\Theta}_{P}$ (or at least an approximation to some of the Pareto set points), should be obtained ${ }^{4}$. With this set the designer has to decide the final solution.

Then, in the MO problem approach the following steps have to be covered:

1.Decision variable codification. To define the decision variable codification ( $\boldsymbol{\theta}$ codification) and the solution search space $(D)$.

2.Objectives selection. To select the objectives to be minimized $(\boldsymbol{J}(\boldsymbol{\theta}))$

3.Optimization process. To obtain a good approximation of $\mathbf{\Theta}_{\mathbf{P}}^{*}$ set with the optimal diffusers in a Pareto optimal sense.

4.Decision making. To choose the optimal diffuser from the $\boldsymbol{\Theta}_{\mathbf{P}}^{*}$.

3 Maximizing an objective can be transformed in a minimization problem, since $\max J_{i}=-\min \left(-J_{i}\right)$.

${ }^{4}$ Notice that $\Theta_{P}^{*}$ is not unique. 


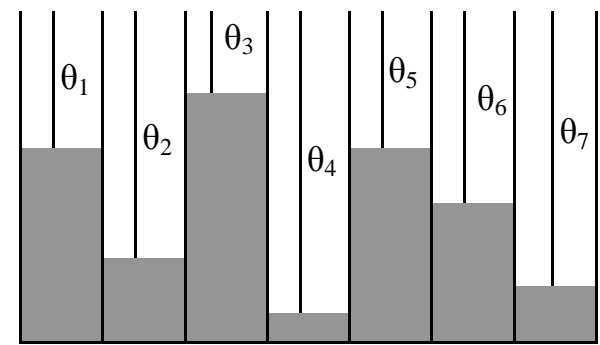

Fig. 3 Profiled diffuser with seven wells. Well width $w=$ $0.9 / 7=0.128 \mathrm{~m}$. Maximum depth of each well $0.32 \mathrm{~m}$.

\subsubsection{Decision variable codification}

The first step should be the decision variable codification considered here as the depth of different-wells. We have assumed in this problem that each one of the wells can take a finite number of depth values, which depend on the precision available in the manufacturing process of the diffuser. In our case, we assume that the minimum step in the depth of a well is $2 \mathrm{~mm}$. As we said above, the maximum depth of a well is $320 \mathrm{~mm}$. Each depth of a well can be varied from 0 to $320 \mathrm{~mm}$ in steps of $2 \mathrm{~mm}$. We have 161 different depths.

Then, we define $\boldsymbol{\theta}$ to characterize the diffusers to be designed as follows:

$\boldsymbol{\theta}=\left[\theta_{1}, \ldots, \theta_{7}\right](7)$

$0 \leq \theta_{i} \leq 160,(1 \leq i \leq 7)$

where $\theta_{\boldsymbol{i}}$ is a integer number that indicates the depth in steps of each one of the seven wells that form the proposed Schroeder diffuser (see Fig. 3).

Using this codification, the depth profile of the diffuser $l(x)$ used in Eq. (1) can be defined as (taking into account that the sample is composed by an array of four diffusers):

$$
\begin{aligned}
& \square \theta_{1} \text { if }|x| \in \quad[0, w[\cup[7 w, 8 w[ \\
& \theta_{2} \text { if }|x| \in[w, 2 w[\cup[8 w, 9 w[ \\
& \theta_{3} \text { if }|x| \in[2 w, 3 w[\cup[9 w, \\
& 10 w[
\end{aligned}
$$

$$
\begin{aligned}
l(x)= & \theta_{4} \text { if }|x| \in[3 w, 4 w[\cup[10 w, 11 w[ \\
& \theta_{5} \text { if }|x| \in[4 w, 5 w[\cup[11 w, 12 w[ \\
& \theta_{6} \text { if }|x| \in[5 w, 6 w[\cup[12 w, 13 w[ \\
& \square \\
& \theta_{7} \text { if }|x| \in[6 w 7 w[\cup[13 w, 14 w[
\end{aligned}
$$

As we have search space dimension of $L=7$ wells and each one can have $\boldsymbol{a}=161$ different depths. The search space encloses:

$$
\boldsymbol{\Theta}^{*}(a, L)=a^{L}=161^{7}=2.8040 \cdot 10^{15}
$$

possible diffusers. Of course, an exhaustive search to obtain the optimal diffuser is not viable. To reduce the searching space, we have used the fact that an important characteristic of the diffuser design is that different diffusers can be equivalents. This characteristic produces a multimodal problem where several points have the same diffusion coefficient values. For instance, if one diffuser is flipped horizontally, the resulting diffusers is equivalent, see Fig. 4 ( $\boldsymbol{\theta}^{a}$ and $\boldsymbol{\theta}^{b}$ diffusers produce the same diffusion coefficient). On the other hand, if we consider a diffuser $\boldsymbol{\theta}^{a}$ with its deepest well $\theta_{3}^{a}$, we can obtain equivalent diffusers $\boldsymbol{\theta}^{c}$ as

$$
\begin{aligned}
\boldsymbol{\theta}^{c} & =\boldsymbol{\theta}^{a}-V[1,1,1,1,1,1,1](10) \\
\gamma & \in\left[1, \ldots,{ }_{3} \theta^{a}\right]
\end{aligned}
$$

If these duplicated combinations are avoided in the codification procedure, a reduction of the search space is achieved and the problem of multimodality is reduced. To eliminate these equivalent diffusers we propose to modify our diffuser codification (Eq. (7)) in such a way that at least one of the seven wells of the diffuser is set to zero ${ }^{5}$.

$$
\begin{aligned}
& \boldsymbol{\theta}=\left[\theta_{1}, \ldots, \theta_{7}\right](11) \\
& 0 \leq \theta_{i} \leq 160,(1 \leq i \leq 7) \\
& \exists i: \theta_{i}=0
\end{aligned}
$$

Then diffusers $\boldsymbol{\theta}^{\boldsymbol{a}}$ and $\boldsymbol{\theta}^{\boldsymbol{b}}$ are not possible with this new codification. Their equivalent diffusers would be $\boldsymbol{\theta}^{c}$ and $\boldsymbol{\theta}^{d}$, respectively (see Fig. 4).

Table 1 shows the different diffusers that can be obtained with this new codification for a seven wells diffuser (taking into account that at least one well is set to 0 ).

In a more general approach, the number of different diffusers, for a $L$ wells diffuser with $a$ possible depths, could be obtained by means of:

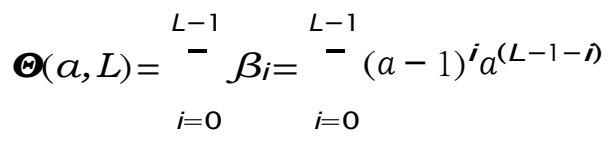

In our case, we obtain $\boldsymbol{\Theta}(161,7)=1.1967 \cdot 10^{14}$, which represents $4.27 \%$ of $\boldsymbol{\Theta}^{*}(161,7)$. That means a huge reduction of the search space.

Besides, each one of these diffusers has its flipped copy. Only the symmetric diffusers has not a flipped

5 This codification is equivatent to eharaeterize the dif-

fusers as a vector of 6 increments. We do not codify the diffusers with increments since it is more difficult to manage the searching space and to implement genetic operators in the MOEA. 

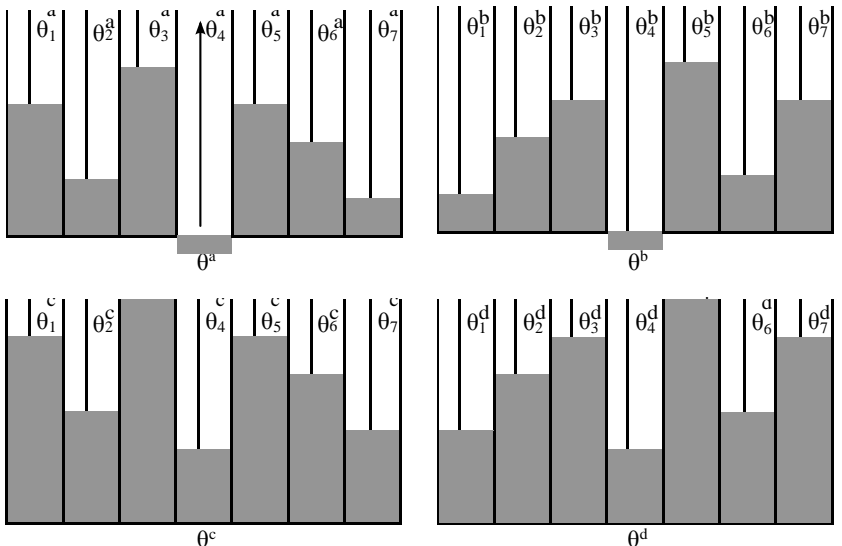

Fig. $4 \boldsymbol{\theta}^{b}$ and $\boldsymbol{\theta}^{d}$ are obtained by flipping horizontally $\boldsymbol{\theta}^{a}$ and $\boldsymbol{\theta}^{5}$, respectively. $\boldsymbol{\theta}^{c}\left(\boldsymbol{\theta}^{d}\right)$ is obtained by subtracting $\theta_{3}^{a}\left(\theta_{5}^{b}\right)$ from $\boldsymbol{\theta}^{a}\left(\boldsymbol{\theta}^{b}\right) . \boldsymbol{\theta}^{a}, \boldsymbol{\theta}^{b}, \boldsymbol{\theta}^{c}$ and $\boldsymbol{\theta}^{d}$ are all equivalent diffusers.

Table 1 Diffusers alternatives with seven wells. $x_{\boldsymbol{j}} \in$ $[0 \ldots a-1]$ and $y_{j} \in[1 \ldots a-1]$, where $a$ is the number of possible depths for each of the $L$ wells. $\beta(i)$ is the number of different combinations.

\begin{tabular}{c|ccccccc|l}
\hline $\boldsymbol{i}$ & $\theta_{1}$ & $\theta_{2}$ & $\theta_{3}$ & $\theta_{4}$ & $\theta_{5}$ & $\theta_{6}$ & $\theta_{7}$ & $\beta(i)$ \\
\hline 0 & 0 & $x_{3}$ & $x_{4}$ & $x_{4}$ & $x_{5}$ & $x_{6}$ & $x_{7}$ & $a^{6}$ \\
1 & $y_{1}$ & 0 & $x_{4}$ & $x_{4}$ & $x_{5}$ & $x_{6}$ & $x_{7}$ & $(a-1) a^{5}$ \\
2 & $y_{1}$ & $y_{2}$ & 0 & $x_{4}$ & $x_{5}$ & $x_{6}$ & $x_{7}$ & $(a-1)^{2} a^{4}$ \\
3 & $y_{1}$ & $y_{2}$ & $y_{3}$ & 0 & $x_{5}$ & $x_{6}$ & $x_{7}$ & $(a-1)^{3} a^{3}$ \\
4 & $y_{1}$ & $y_{2}$ & $y_{3}$ & $y_{4}$ & 0 & $x_{6}$ & $x_{7}$ & $(a-1)^{4} a^{2}$ \\
5 & $y_{1}$ & $y_{2}$ & $y_{3}$ & $y_{4}$ & $y_{5}$ & 0 & $x_{7}$ & $(a-1)^{5} a$ \\
6 & $y_{1}$ & $y_{2}$ & $y_{3}$ & $y_{4}$ & $y_{5}$ & $y_{6}$ & 0 & $(a-1)^{6}$ \\
\hline
\end{tabular}

Table 2 Symmetric diffusers alternatives with seven wells. $x_{\boldsymbol{j}} \in[0 \ldots a-1]$ and $y_{\boldsymbol{j}} \in[1 \ldots a-1]$, where $a$ is the number of possible depths for each of the $L$ wells. $\beta(i)$ is the number of different combinations.

\begin{tabular}{c|ccccccc|l}
\hline $\boldsymbol{i}$ & $\theta_{1}$ & $\theta_{2}$ & $\theta_{3}$ & $\theta_{4}$ & $\theta_{5}$ & $\theta_{6}$ & $\theta_{7}$ & $\beta^{s}(i)$ \\
\hline 0 & 0 & $x_{2}$ & $x_{3}$ & $x_{4}$ & $x_{3}$ & $x_{2}$ & 0 & $a^{3}$ \\
1 & $y_{1}$ & 0 & $x_{3}$ & $x_{4}$ & $x_{3}$ & 0 & $y_{1}$ & $(a-1) a^{2}$ \\
2 & $y_{1}$ & $y_{2}$ & 0 & $x_{4}$ & 0 & $y_{2}$ & $y_{1}$ & $(a-1)^{2} a$ \\
3 & $y_{1}$ & $y_{2}$ & $y_{3}$ & 0 & $y_{3}$ & $y_{2}$ & $y_{1}$ & $(a-1)^{3}$ \\
\hline
\end{tabular}

copy since it is itself. For a $L$ dimensional diffuser ( $L$ an even integer number), the number of symmetric diffusers is obtained as (see Table 2):

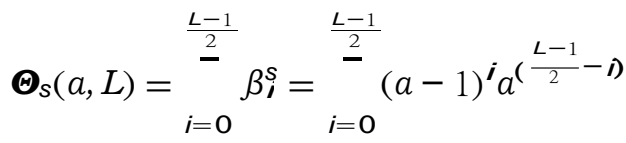

The number of non-symmetric diffusers is:

$\boldsymbol{\Theta}_{n s}(a, L)=\boldsymbol{\Theta}(a, L)-\boldsymbol{\Theta}_{s}(a, L)$

Therefore, the total number of different diffusers can be calculated as:

$$
\begin{aligned}
\boldsymbol{\Theta}_{t}(a, L) & =\frac{\boldsymbol{\Theta}(a, L)-\boldsymbol{\Theta}_{s}(a, L)}{{ }^{2} \boldsymbol{\Theta}_{s}(a, L)} \\
& =\frac{\boldsymbol{\Theta}(a, L)}{2}(a, L)(15)
\end{aligned}
$$

In our case study $\boldsymbol{\Theta}_{t}(161,7)=5.9833 \cdot 10^{13}$, which is the approximately $50 \%$ of $\boldsymbol{\Theta}(161,7)$ and $2.13 \%$ of $\boldsymbol{\Theta}^{*}(161,7)$. Therefore the number of possible diffusers has been reduced drastically but, even that reduction, an exhaustive search is not affordable (search space still remains vast and an optimization algorithm is necessary). Then to avoid flipped diffusers, the following con-
straints have to be satisfied:

$$
\begin{array}{r}
\left(\theta_{1}<\theta_{7}\right) \vee \\
\left(\theta_{1}=\theta_{7} \wedge \theta_{2}<\theta_{6}\right) \vee \\
\left(\theta_{1}=\theta_{7} \wedge \theta_{2}=\theta_{6} \wedge \theta_{3} \leq \theta_{5}\right)
\end{array}
$$

In summary codification is guided with Eq. (11) and Eq. (16) to reduce the search space and to avoid multimodality.

\subsubsection{Objectives selection}

An important step in the multiobjective approach is an adequate selection of the objectives of the optimization process (the components of $\boldsymbol{J}(\boldsymbol{\theta})$ ). The ideal choice has to be closely related to the design preferences. As mentioned earlier, our main objective is to maximize $d_{j}$ in the range of the selected frequencies.

A classical alternative is to minimize an objective function that will be equivalent to maximize $d_{j}$. For instance:

$$
J_{1}(\boldsymbol{\theta})=1-\bar{d}=1-{ }_{j=1}^{6} \frac{d_{j}(\boldsymbol{\theta})}{6}
$$

where $\bar{d}$ represents the mean normalized diffusion coefficient for the one third-octave bands selected.

Minimization of Eq. (17) could produce diffusers with good performance in average, but their frequency response could present high dispersion. To reduce this effect another objective have to be introduced, the standard deviation. This second objective function is defined as:

$J_{2}(\boldsymbol{\theta})=\frac{-\overline{\boldsymbol{j}_{j=1}\left(\bar{d}-d_{j}(\boldsymbol{\theta})\right)^{2}}}{5}$ 
Table 3 Preferences ranges in GPP.

\begin{tabular}{|c|c|c|c|}
\hline & Desirable & Tolerable & Undesirable \\
\hline $\begin{array}{l}J_{1}(\theta) \\
J_{2}(\theta)\end{array}$ & 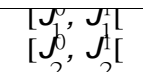 & $\begin{array}{l}{\left[J_{1}^{1}, J_{1}^{2}[\right.} \\
{\left[J_{2}^{1}, J_{2}^{2}[\right.}\end{array}$ & $\begin{array}{l}J_{1}^{2}, J_{1}[ \\
{\left[J_{2}^{2}, J_{2}^{3}[\right.}\end{array}$ \\
\hline
\end{tabular}

Both objectives could be in conflict and can be treated with a multiobjective approach instead of merging them in an single aggregate objective (a common way to solve a multiobjective problem).

Variations changing the selected frequencies in Eq. (17) could be used as alternative or additional objectives.

\subsubsection{Optimization process}

For our optimization problem, we will use an elitist multi-objective evolutionary algorithm, based on the concept of s-dominance [10], named ev-MOGA [5, 6]. evMOGA uses a real codification for the searching space, so that we need to modify ev-MOGA to implement the diffusers codification presented above. Besides some modifications are required in the genetic operators of ev-MOGA to avoid different equivalent diffusers in the evolution process. These issues will be treated in section 3.

\subsubsection{Decision making}

The final step is the analysis of the Pareto approximation obtained to select the preferred solution. Several graphical tools can be used [20], specially when

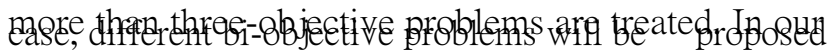
and scatter plots will be used. This graphical represen-

tation of the Pareto front help the designers to better adjust their preferences and to choose the final solution.

An intuitive way to introduce preferences into the decision process is the Physical Programming (GPP)

[16]. The designers have to introduce their preferences by means of a table with preferred ranges of performances. Each one of these ranges could be labeled to facilitate the interpretability. The GPP combines all this information to produce a value that could rank the solutions of the Pareto front. The GPP is based on weighting equally each range for all the objectives and applying " One vs. Others Criteria Rule (OVO-rule)" [13] (minimizing the worst value, so a $[T, T]$ solution is preferred over $[D, U]$ one).

For a two objectives MOP $\left(J_{1}(\boldsymbol{\theta}), J_{2}(\boldsymbol{\theta})\right)$ and three interval ranges of preferences, labeled as "Desirable" (D), "Tolerable" (T) and "Undesirable" (U), the designer has to fulfill the Table 3.
It is important to remark that these ranges are in physical units of the objectives, being understandable by the DM.

\section{3 ev-MOGA for diffusers optimization}

ev-MOGA uses three populations $\left(P_{t}\right.$ with fixed size $n_{P}, A_{t}$ with size $n_{A}$ and $t t_{t}$ with fixed size $\left.n_{G}\right)$ in the optimization process ( $t$ is the algorithm iteration). $A_{t}$ promotes convergence of the solutions toward Pareto front $\boldsymbol{J}\left(\boldsymbol{\Theta}_{P}\right)$ in a smart distributed way. $A_{t}$ is an archive, where the solution of the optimization problem $\boldsymbol{\Theta}^{*}{ }_{P}$ is stored. Its size can change but its maximum size is bounded; $P_{t}$ is the main population, it explores the searching space and converges to the Pareto front. During the evolutionary process this population is updated only with dominance considerations. $t t_{t}$ is an auxiliary population which maintain temporary solutions created by means of genetic operators. Solutions in $t t_{t}$ are used to update $P_{t}$ and $A_{t}$.

To achieve an smart distributed Pareto front $\boldsymbol{J}\left(\boldsymbol{\Theta}_{P}^{*}\right)$ only solutions with significant trade-off are kept in $A_{t}$. To do that, $\boldsymbol{s}$-dominance is used and the objective space is split into a fixed number of boxes $n$ box. This grid preserves the diversity of $\boldsymbol{J}\left(\boldsymbol{\Theta}^{*}\right)_{p}$ since each box can only be occupied by one solution. When in the update process of $A_{t}$, two solutions are in the same box, the solution that prevails will be the one that is closest to the center of the box.

Figure 5 shows how $\boldsymbol{J}\left(\boldsymbol{\Theta}_{p}^{*}\right)$ could be obtained by applying s-dominance concept for an example with two objectives, when $n$ box $=10$ is used. The vialues $s_{1}$ and $S_{2}$ depend on the limits of the front $J_{\text {min }} \max$ and $J^{\max } \quad 1, J_{2}, J_{1}$ 2 , which are adjusted dynamically in each algorithm iteration.

The distribution of solutions along the Pareto front depends on the selected grid.

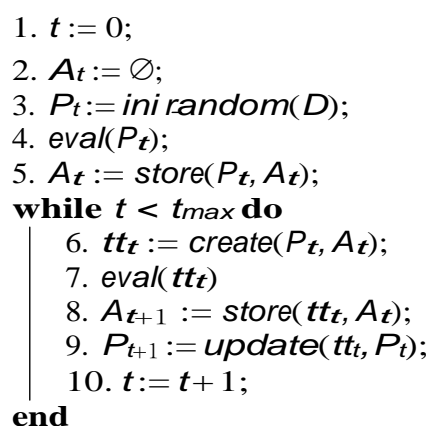

Algorithm 1: Pseudocode of ev-MOGA.

Algorithm 1 shows the pseudocode of ev-MOGA. 


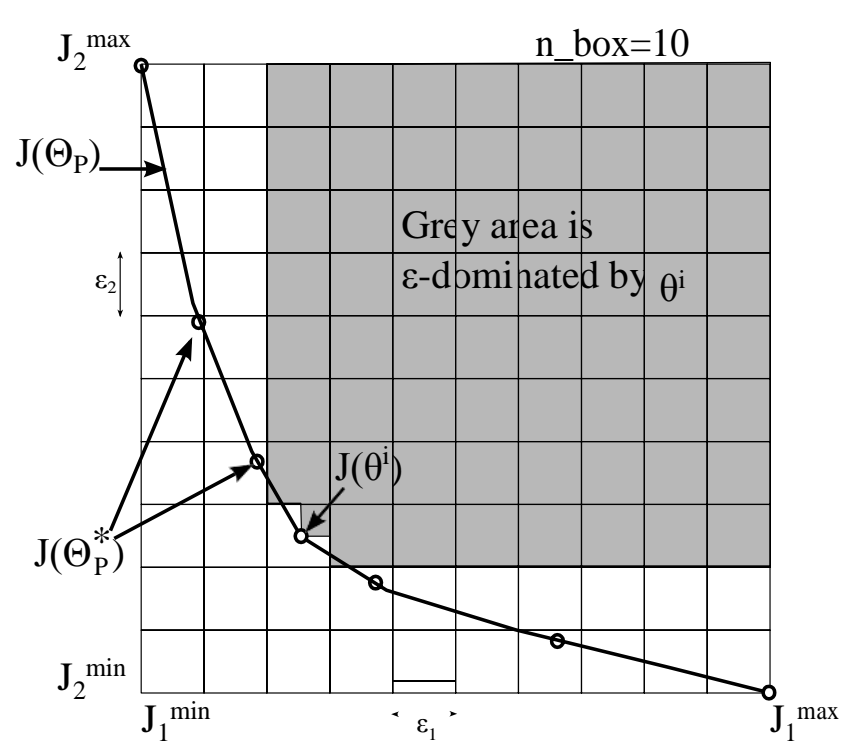

Fig. 5 The concept of $s$-dominance in a bi-objective problem. $\boldsymbol{J}_{\left(\boldsymbol{\Theta}^{*}\right)}$ is the smart characterization of the Pareto $\boldsymbol{s}_{1}=\left(\boldsymbol{J}^{\max }-\mathrm{J}_{\min } \mathrm{J}_{1}, \mathrm{~J}_{2}\right.$ max $\min$ $s_{1}=\left(J_{1}\right) / n-b o x, s_{2}=\left(J_{2}-J_{2}\right) / n$ box box widths; and $n$ box, number of boxes for each dimension.

Line 3. $P_{0}$ is initialized with $n_{P}$ diffusers that are randomly generated from diffusers alternatives of Table 1 (with the same probability). If one diffuser does not fulfill Eq. (16) the diffusers is horizontally flipped.

Lines 4 and 7.Function eval calculates the objective vector $\boldsymbol{J}(\boldsymbol{\theta})$ for each diffuser $\boldsymbol{\theta}$ in $P_{t}$ (line 4) or in $t t_{t}$ (line 7).

Line 5.Function store checks individuals in $P_{t}$ (line 5) or $t t_{t}$ (line 8 ) that might be included in the archive At.

1.Non-dominated $P_{t} \cup A_{t}$ (line 5) or $t t_{t} \cup A_{t}$ (line 8) diffusers are detected, $\boldsymbol{\Theta}_{N D}$.

2.Pareto front limits $\boldsymbol{J}_{\boldsymbol{i}}^{\text {max }}$ and $\boldsymbol{J}_{\boldsymbol{i}}^{\text {min }}$ are calculated from $\mathbf{J}(\boldsymbol{\theta}), \forall \boldsymbol{\theta} \in \boldsymbol{\Theta}_{N D}$.

3.Individuals in $\boldsymbol{\Theta}_{N D}$ are analyzed and those that are not $s$-dominated by individuals in $A(t)$, will be included in $A(t)$.

Line 6. With each iteration, the function create creates $t t_{t}$ as follows:

1. Two individuals are randomly selected, $\boldsymbol{\theta}^{P}$ from $P_{t}$ and $\boldsymbol{\theta}^{A}$ from $A_{t}$.

2.A random number $u \in[0 \ldots 1]$ is generated.

3.If $u>P_{c / m}$ (probability of crossover/mutation), $\boldsymbol{\theta}^{P}$ and $\boldsymbol{\theta}^{A}$ are crossed over by means of the onepoint recombination technique.

4.If $u \leq P_{c / m}, \boldsymbol{\theta}^{P}$ and $\boldsymbol{\theta}^{A}$ are mutated using random mutation with Gaussian distribution [5] and then included in $t t_{t}$.
5.Crossover and mutation operators have been modified to avoid duplicities (satisfying Eq. (11) and Eq. (16)).

This procedure is repeated $N i n d_{G} / 2$ times until $t t_{t}$ is filled.

Line 10.Each diffuser $\boldsymbol{\theta}^{\boldsymbol{g}}$ in $\boldsymbol{t} t_{t}$ is compared with a randomly selected diffusers $\boldsymbol{\theta}^{P}$ in $P_{t}$. If $\boldsymbol{\theta}^{\boldsymbol{G}}$ dominates $\boldsymbol{\theta}^{P}, \boldsymbol{\theta}^{G}$ replaces $\boldsymbol{\theta}^{P}$ in $P_{t}$

At the end, diffusers in $A_{t}$ compose $\boldsymbol{\Theta}_{P}^{*}$, the smart approximation to the Pareto front.

\section{Multiobjective design of proftled diffusers. Approaches}

Three different multiobjective problems will be proposed in order to obtain diffusers with different characteristics.

- MOP1: in which the mean normalized diffusion coefficient $\bar{d}$ for the bands of frequencies $\left[f_{1}, f_{2}, \cdots f_{6}\right]$ is maximized (Eq. (17)) and its standard desviation is minimized (Eq. (18)).

- MOP2: where $\boldsymbol{d}$ is maximized for two different bands of frequencies, low $\left[f_{1}, f_{2}, f_{3}\right]$ and high $\left[f_{4}, f_{5}, f_{6}\right]$ frequencies. These objectives are obtained from Eq. (17) for each range of frequencies.

- MOP3: where $\vec{a}$ is maximized for $\left[f_{1}, f_{2}, \cdots f_{6}\right]$ frequencies (Eq. (17)) and for the $f_{1}$ frequency in particular.

In MOP1, MOP2 and MOP3, the same ev-MOGA configuration has been tuned. The ev-MOGA parameters are set as:

$-n_{P}=30000, n_{G}=8$.

$-n$ box $=40$.

$-P_{c / m}=0.5$. $\sigma_{\text {ini }}=10 \%$ and $\sigma_{\text {fin }}=1 \%[5]$.

$-t_{\text {max }}=50000$.

To reduce the random effect of the MOEA algorithms, the ev-MOGA algorithm is run 10 times and all the resulting Pareto fronts are merged and resampled with the grid used in ev-MOGA.

\subsection{MOP1}

In this MOP the objective function vector is the following:

$$
\boldsymbol{J}(\boldsymbol{\theta})=\left[J_{1}(\boldsymbol{\theta}), J_{2}(\boldsymbol{\theta})\right]
$$

where

$J_{1}(\boldsymbol{\theta})=1-d, J_{2}(\boldsymbol{\theta})=\frac{-\overline{-\xi_{=1}\left(d-d_{j}(\boldsymbol{\theta})\right)^{2}}}{5}$ 

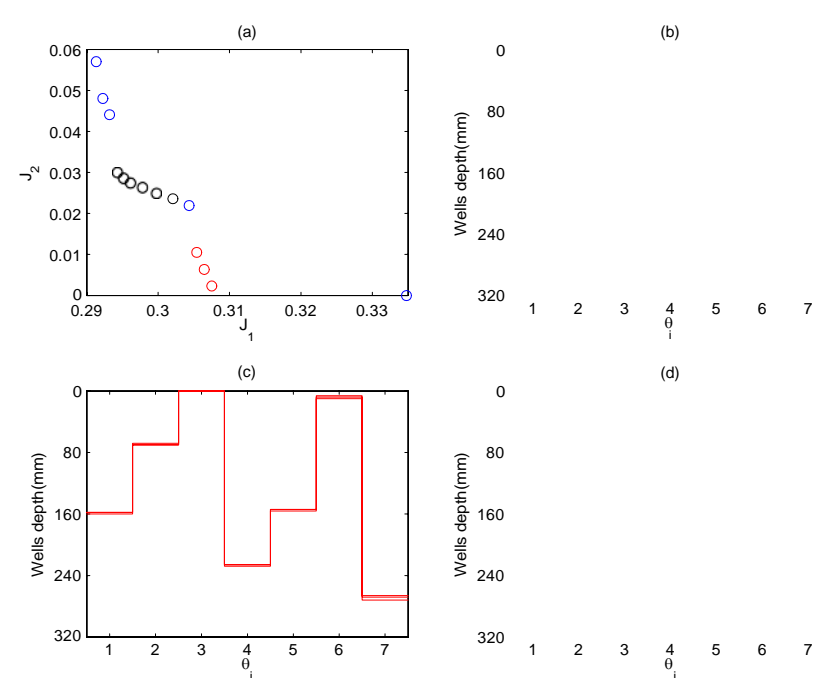

Fig. 6 (a) Pareto front and (b-d) Pareto set for MOP1.

$$
\bar{d}=\underbrace{-6}_{\boldsymbol{j}=1} \frac{\mathrm{d}_{i}(\boldsymbol{\theta})}{6}
$$

Fig. 6.a shows the Pareto front approximation $\boldsymbol{J}\left(\boldsymbol{\Theta}_{\text {MOP }}^{*}\right)$ obtained in the optimization process. The trivial solution $\boldsymbol{\theta}=[0,0,0,0,0,0,0]$ produces $\boldsymbol{J}(\boldsymbol{\theta})=$ $[1,0]$. To avoid this undesired solution $J_{1}$ is modified saturating every value $J_{1}(\boldsymbol{\theta})<0.001$ to zero. The resulting Pareto front approximation is disjoint and non convex. This type of fronts is not attainable with a classical multiobjective technique based on solving the single-objective problem that results from a weighted aggregation of both objectives.

Analyzing the result, the Pareto front is in the ranges of $J_{1} \in[0.291 \ldots 0.335]$ and $J_{2} \in[0 \ldots 0.06]$. It is easy to see that the Pareto set has only three different types of solutions. $\boldsymbol{\Theta}_{M O P}^{*}$ has been divided in three subsets of similar diffusers (see Fig. 6.b, Fig.6.c and Fig.6.d).

Fig. 4.1 shows the frequency response of the normalized diffusion coefficient for each diffuser in Pareto set QMOP 1. It is possible to find diffusers that present high values for $\bar{d}$ (low $J_{1}$ ) but with major dispersion $\left(J_{2}\right)$. By contrast diffusers with low dispersion produce low values for $\bar{d}$ (high $J_{1}$ ). It is noteworthy the performances obtained by the subset of diffusers of Fig. 6.b. This subset (blue point in the Pareto front) presents similar diffusers but produces very different performance since they cover the extremes of the Pareto front and one of them is equally balance between $J_{1}$ and $J_{2}$ (centered in the Pareto front). That means a slight variation of this configuration produces quite different performance showing how sensitive the design of the diffusers is for $J_{1}$ and $J_{2}$ objectives.

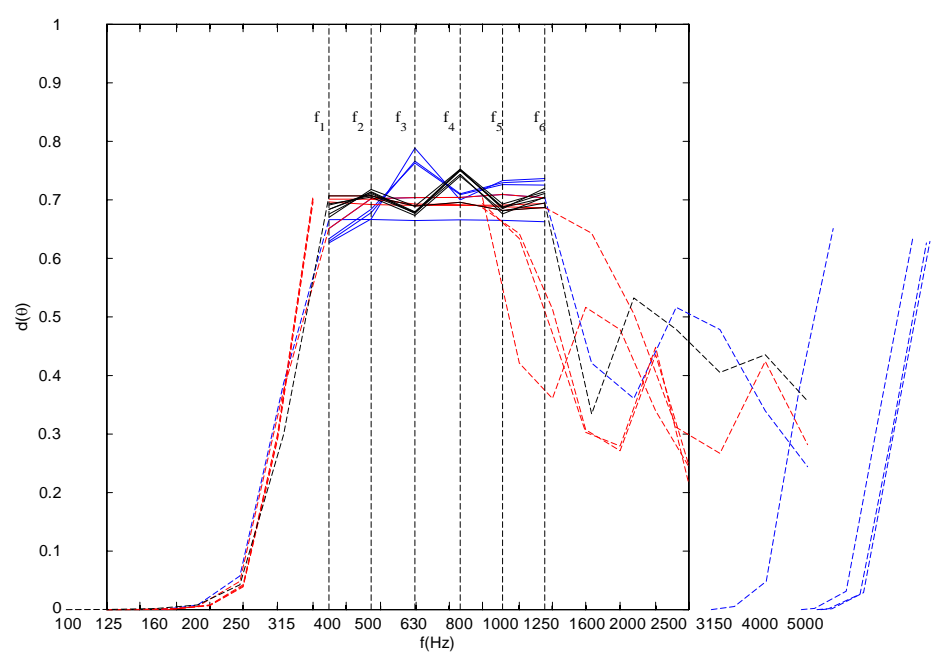

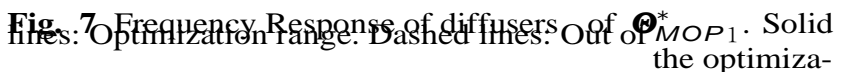
tion range. Notice that frequencies over $1332 \mathrm{~Hz}$ are over the limit of validity of the Fraunhofer theory.

This sensitivity induces the designer to add some new desired performances related to the robustness of the diffusers in the manufacturing process. This new performance could be included in the design process as additional objectives requiring the definition of a new expression to evaluate it and to redo the optimization process. In order to avoid more complex fronts (threeobjective problem), the robustness requirement is introduced in the decision making step.

The way to evaluate the robustness is through the degradation of the performances when the solution is subjected to a slight variation. For each $\boldsymbol{\theta}^{*} \in \boldsymbol{\Theta}_{M O P}$, a set $\boldsymbol{\Theta}_{R}\left(\boldsymbol{\theta}^{*}\right)$ with $6^{3}=216$ diffusers is generated. $\boldsymbol{\Theta}_{R}\left(\boldsymbol{\theta}^{*}\right)$ contains small variations of $\boldsymbol{\theta}^{*}$ produced by changing the depth of its well (adding and subtracting one step) except for the well whose value is zero. The value for each one of these variations $\boldsymbol{J}\left(\boldsymbol{\Theta}_{R}\right)$ is calculated and its envelop represented in Fig. 4.1 next to the Pareto front. So it is possible to see the degradation in the performance of each diffuser belonging to the Pareto Front.

We note that diffusers belonging to subset of Fig.6.b (blue one) are less robust than diffusers of the subset in Fig.6.c (black) due to the areas of the envelopes are higher. Then it seems reasonable to select the final diffuser from the black ones. To select the final solution we will use the GPP methodology. The ranges of preferences chosen ${ }^{7}$ are shown in Table 4.

By applying these ranges (see Fig. 4.1) we get three diffusers as $[T, U]$, five as $[T, T]$, two as $[U, T]$ and four as $[U, D]$. Among the five $[T, T]$ diffusers we choose the

7 Based in the analyses of the Pareto front of Fig. 6.a. 


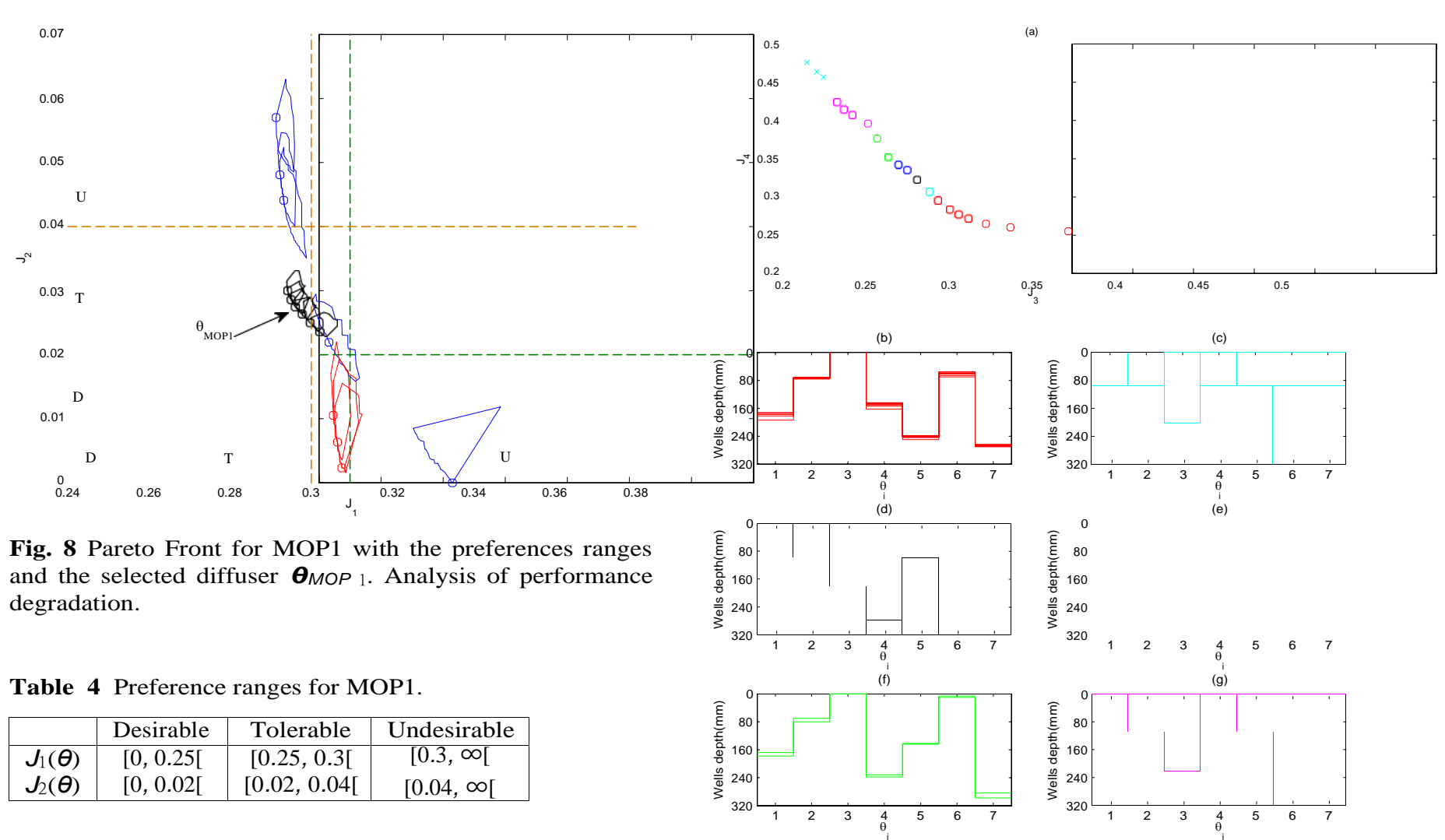

diffusers nearest to the ideal point of the $[T, T]$ area $\boldsymbol{J}=[0.25,0.02]$. Then we get:

Fig. 9 (a) Pareto front and (b-g) Pareto set for MOP2.

$\boldsymbol{\theta}_{M O P 1}=[100,0,184,272,80,292,220] \mathrm{mm}$

$\left[J_{1}\left(\boldsymbol{\theta}_{M O P 1}\right), J_{2}\left(\boldsymbol{\theta}_{M O P 1}\right)\right]=[0.2961,0.0275]$

Remark that $\theta_{M O P} 1$ (remarked in Fig. 4.1) is in a non convex part of the Pareto front, so it couldn' $t$ be found by a classical aggregation method based on weighting.

\subsection{MOP2}

In order to evaluate other possibilities, different objectives are set. This second problem tries to find solutions based on optimizing average diffusion coefficient in two frequency bands. In this MOP the new objective vector is:

$$
\boldsymbol{J}(\boldsymbol{\theta})=\left[J_{3}(\boldsymbol{\theta}), J_{4}(\boldsymbol{\theta})\right]
$$

where

$$
J_{3}(\boldsymbol{\theta})=1-\underbrace{3}_{\boldsymbol{j}=1} \frac{d(\boldsymbol{\theta})}{3}, J_{4}(\boldsymbol{\theta})=1-\underbrace{\frac{6}{\boldsymbol{j}}}_{\boldsymbol{j}=4} \frac{\mathbf{d}(\boldsymbol{\theta})}{3}
$$

Fig. 9.a shows the Pareto front $\boldsymbol{J}\left(\boldsymbol{\Theta}^{*}{ }_{\text {MOP } 2)}\right)$. The Pareto front covers ranges $J_{3} \in[0.21 \ldots 0.46]$ and $J_{4} \in$
$[0.24 \ldots 0.48] . J_{3}$ includes $f_{1}, f_{2}$ and $f_{3}$ frequencies and $J_{4}$ includes $f_{4}, f_{5}$ and $f_{6}$ ones. $J_{3}$ values are lower than $J_{4}$ ones, it is easier to obtain diffusers with better performance for low frequencies $\left(J_{3}\right)$ than for high $\left(J_{4}\right)$.

The Pareto set $\boldsymbol{\Theta}^{*}{ }_{N D P} 2$ could be clustered by similarity in different groups of diffusers. The $\boldsymbol{\Theta}_{M}^{*} O_{8} 2$ has been divided in several subsets of similar diffusers ${ }^{8}$ (see Fig. 9.b, Fig.9.c, Fig. 9.d, Fig. 9.e, Fig. 9.f and Fig. 9.g).

In Fig. 4.2 the frequency response of the normalized diffusion coefficient $d$ for the diffusers in Pareto set $\boldsymbol{\Theta}_{\text {MOP } 2}$ is presented. We can see the trade-off between the different diffusers since the diffusers with high values of $d$ for low frequencies present minor values $d$ for low frequencies and vice versa.

The decision making process is assisted by the analysis of the robustness of the diffusers in $\boldsymbol{\Theta}_{M O P}^{*}$. The same study presented in MOP1 subsection is repeated here. Fig. 4.2 shows that solutions in the Pareto set are quite robust since the loss of performance is small and much lower than in MOP1.

\footnotetext{
${ }^{8}$ The subsets corresponding to the extreme of the Pareto front (represented by blue 'x' in Fig. 9.a) are not represented in Fig. 9 since they are less interesting for the decision making.
} 

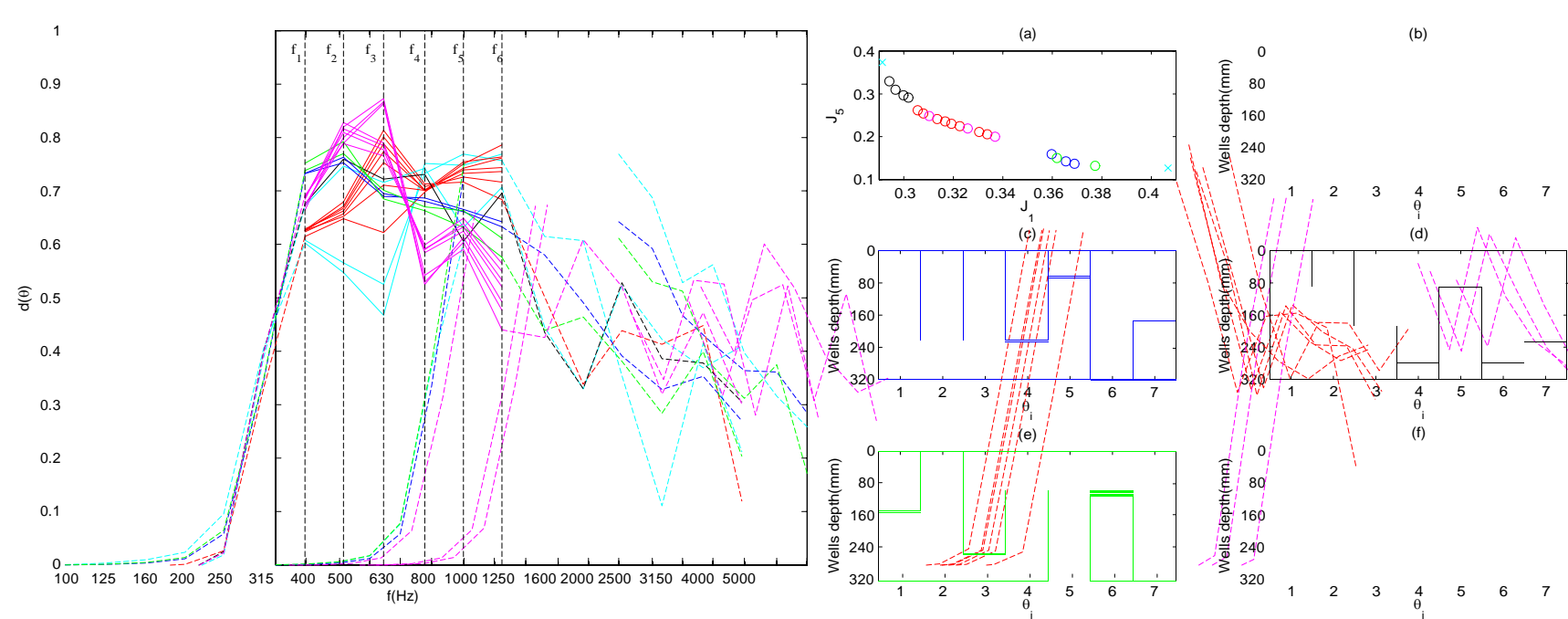

Fig. 10 Frequency Response for MOP2. Solid lines: Optimization range. Dashed lines: Out of the optimization range. Notice that frequencies over $1332 \mathrm{~Hz}$ are over the limit of validity of the Fraunhofer theory.

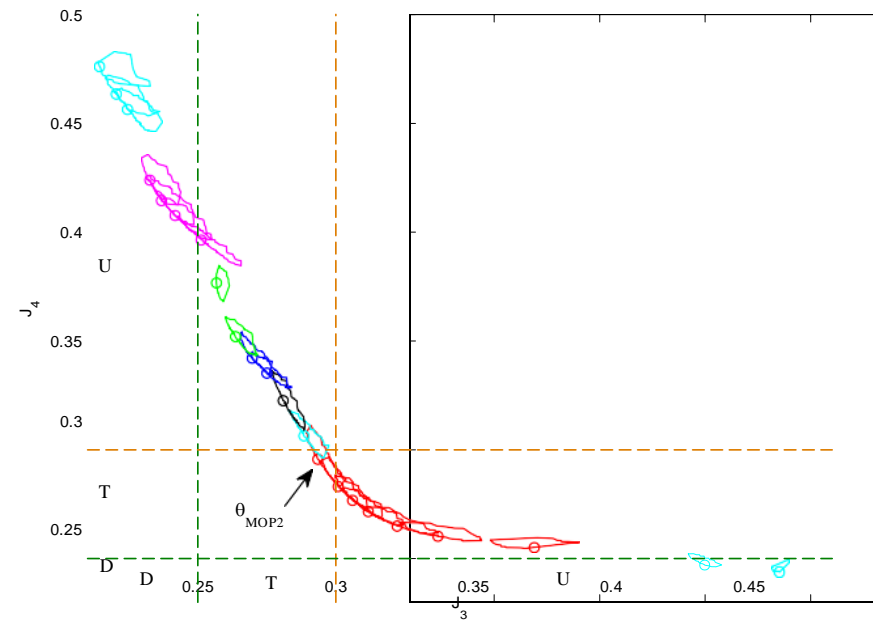

Fig. 11 Pareto Front for MOP2 with the preferences ranges

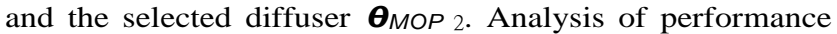
degradation.

Table 5 Preference ranges for MOP2.

\begin{tabular}{|c|c|c|c|}
\hline & Desirable & Tolerable & Undesirable \\
\hline$J_{3}(\theta)$ & {$[0,0.25[$} & {$[0.25,0.3[$} & {$[0.3, \infty[$} \\
$J_{4}(\theta)$ & {$[0,0.25[$} & {$[0.25,0.3[$} & {$[0.3, \infty[$} \\
\hline
\end{tabular}

To select the final solution, by means of GPP methodology, we keep the range of preferences chosen for $J_{1}$ in $M O P 1$. Then we use the preferences shown in Table 5.

By applying these ranges we get six diffusers as $[D, U]$, two as $[U, D]$, seven as $[T, U]$, six as $[U, T]$ and only one as $[T, T]$. Then we get the latter one which belongs to the subset represented in Fig. 9.b. Fig. 11 remaks $\boldsymbol{\theta}_{\text {MOP } 2}$ in the Pareto front.
Fig. 12 (a) Pareto front and (b-f) Pareto set for MOP3.

\subsection{MOP3}

In this MOP, the objective vector is built with $J_{1}$ of MOP1 (related to average diffusion coefficient) and an indicator of the diffusion coefficient at frequency $f_{1}$ :

$\boldsymbol{J}(\boldsymbol{\theta})=\left[J_{1}(\boldsymbol{\theta}), J_{5}(\boldsymbol{\theta})\right]$

where $J_{1}(\boldsymbol{\theta})$ is the same as in MOP1 and $J_{5}(\boldsymbol{\theta})=1-d_{1}(\boldsymbol{\theta})$

where $d_{1}(\boldsymbol{\theta})$ is the normalized diffusion coefficient for the low frequency considered, $f_{1}$.

Fig. 4.3.a represents the Pareto front $\boldsymbol{J}\left(\boldsymbol{\Theta}_{\text {MOP 3 }}^{*}\right)$ which covers ranges $J_{1} \in[0.291 \ldots 0.334]$ and $J_{5} \in$ $[0.127 \ldots 0.373]$. As expected, we see that it is easier to obtain diffusers with better performance for only one frequency $\left(J_{5}\right)$ than for a range of frequencies $\left(J_{1}\right)$ due to the values of $J_{5}$ are lower than $J_{1}$. As in MOP2, the @MOP 3 has been divided in several subsets according to its similarity ${ }^{9}$ (see Fig. 4.3.b, Fig.4.3.c, Fig. 4.3.d, Fig. 4.3.e and Fig. 4.3.f).

Fig. 4.3 shows the frequency response for the diffusers in Pareto set $\boldsymbol{\Theta}_{\text {MOP } 3}^{*}$ and Fig. 4.3 analyses the robustness of such diffusers.

\footnotetext{
${ }^{9}$ The subsets corresponding to the extreme of the Pareto front (represented by blue ' $x$ ' in Fig. 4.3.a) are not represented.
} 


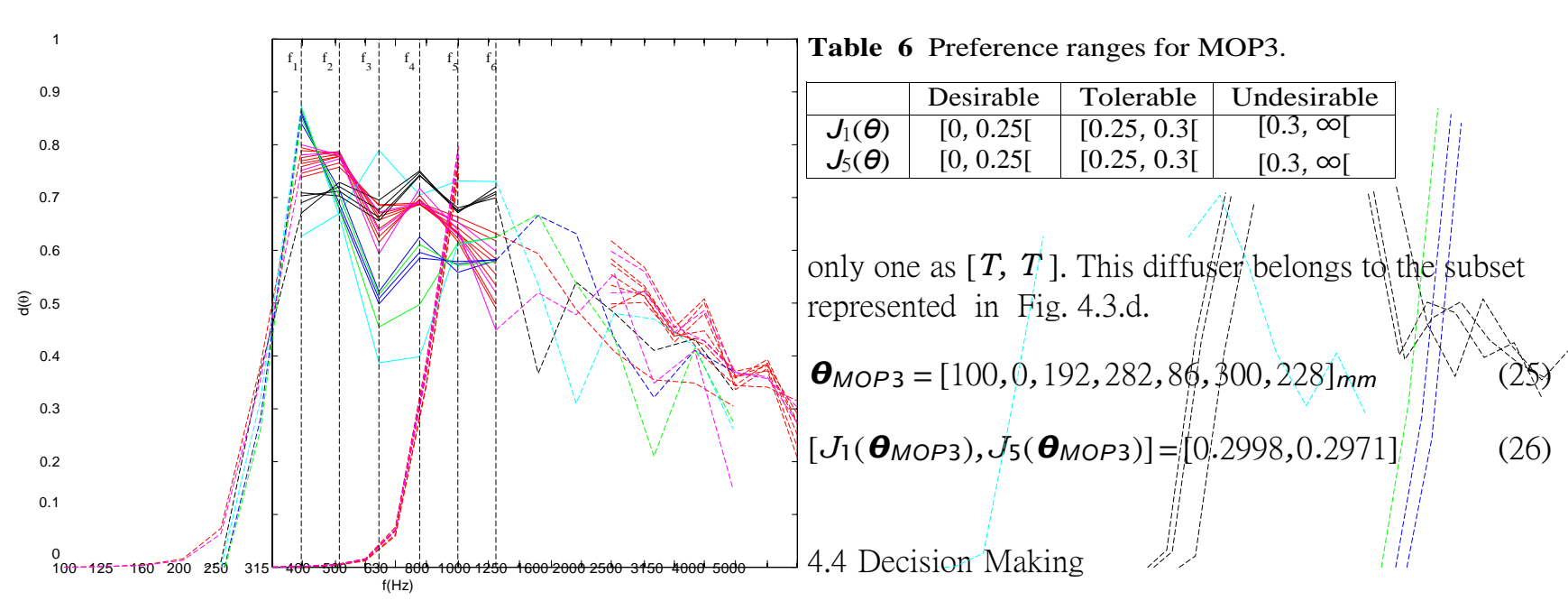

Fig. 13 Frequency Response for MOP3. Solid lines: Optimization range. Dashed lines: Out of the optimization range. Notice that frequencies over $1332 \mathrm{~Hz}$ are over the limit of validity of the Fraunhofer theory.

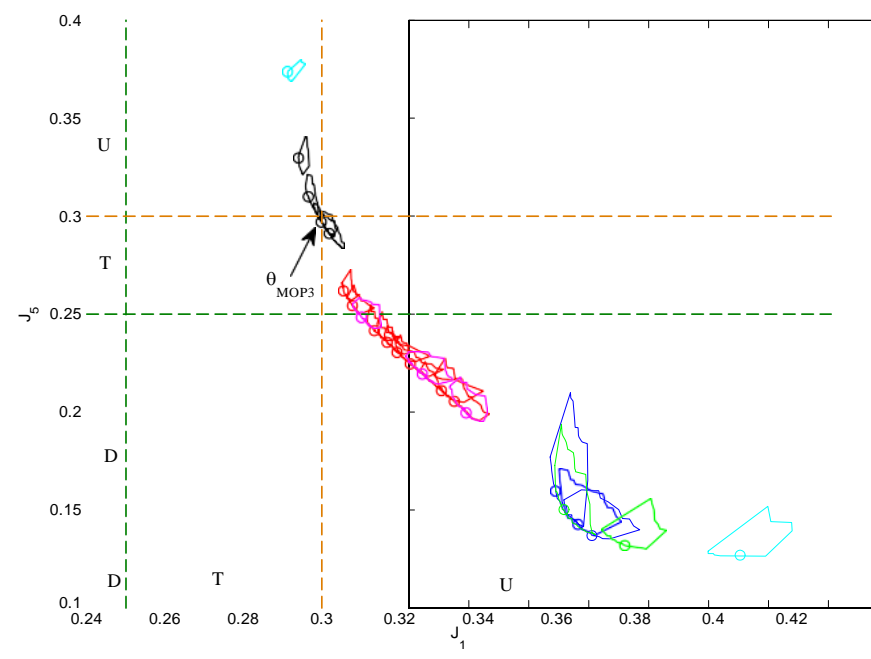

Fig. 14 Pareto Front for MOP3. Analysis of performance degradation.

We notice that we can find diffusers with good performance in $J_{5}$ (values near 0.1 ) but in contrast they produce very bad performance (undesirable if we consider the range presented in MOP2) in $J_{1}$. So we know that it is possible to improve considerably the normalized diffusion coefficient $d$ for one frequency but producing a degradation of the response at other frequencies. Besides diffusers with good performance in $J_{5}$ are less robust that the other (see Fig. 4.3, the envelopes have a higher area).

If we use the same range of preferences presented in MOP2 for the objectives in MOP3 (see Table 6), there is no diffusers as $[D, D],[D, T]$ or $[T, D]$; and there is
As it is shown above, depending on the objectives of the problem the set of solutions could be different and this fact requires a final decision based on designer preferences. In the previous section a decision making process has been performed for each problem separately even taking in to account the particular robustness characteristics. For this final decision process all the previous results will be considered to obtain a solution more reliable according to the designer preferences.

In order to select a final diffuser, the performances of the Pareto sets $\boldsymbol{\Theta}_{M O P_{1}}^{*}, \boldsymbol{\Theta}_{M O P_{2}}^{*}$ and $\boldsymbol{\Theta}_{M O P}^{*}$ are compared among them and against a classical QR diffuser sequence $[0,1,4,2,2,4,1]$ (see Fig. 1) designed using our maximum depth and width for each well, which we will appoint as $\boldsymbol{\theta}_{Q R}$ (see Eq. (27)).

$\boldsymbol{\theta}_{Q R}=[0,80,320,160,160,320,80]_{m m}$

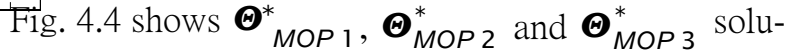
tions in MOP1 context $\left(J_{1}\right.$ versus $\left.J_{2}\right)$, Fig. 16 in MOP2 $\left(J_{3}\right.$ versus $\left.J_{4}\right)$ and Fig. 4.4 in MOP3 $\left(J_{1}\right.$ versus $\left.J_{5}\right)$. Besides, in these figures, the preference ranges for each objective and the performance of the $\boldsymbol{\theta}_{M O P}, \boldsymbol{\theta}_{M O P}$, $\boldsymbol{\theta}_{M O P} 3$ and $\boldsymbol{\theta}_{Q R}$ diffusers are pointed. In Fig. 4.4 the different frequency response of the selected diffusers is shown.

If we compare these four solutions (see Table 7) we realize that, except the $\boldsymbol{\theta}_{Q R}$, all of them present a balanced set of performances. As expected, the performance of $\boldsymbol{\theta}_{Q R}$ is worse and highlight the need for an optimization process to get better performance Schroeder diffusers. $\boldsymbol{\theta}_{M O P} 2$ has good values for $J_{1}, J_{3}$ and $J_{4}$ but worse for $J_{2}$ and $J_{5}$. $\boldsymbol{\theta}_{M O P} 1$ and $\boldsymbol{\theta}_{M O P} 3$ are more bal-

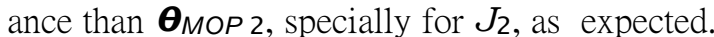

Comparing the selected diffusers, it seems that $\boldsymbol{\theta}_{M O P 1}$ and $\boldsymbol{\theta}_{M O P} 3$ have the same structure, belonging to black subsets (Fig. 6.d and Fig. 4.3.d, respectively). It is not 


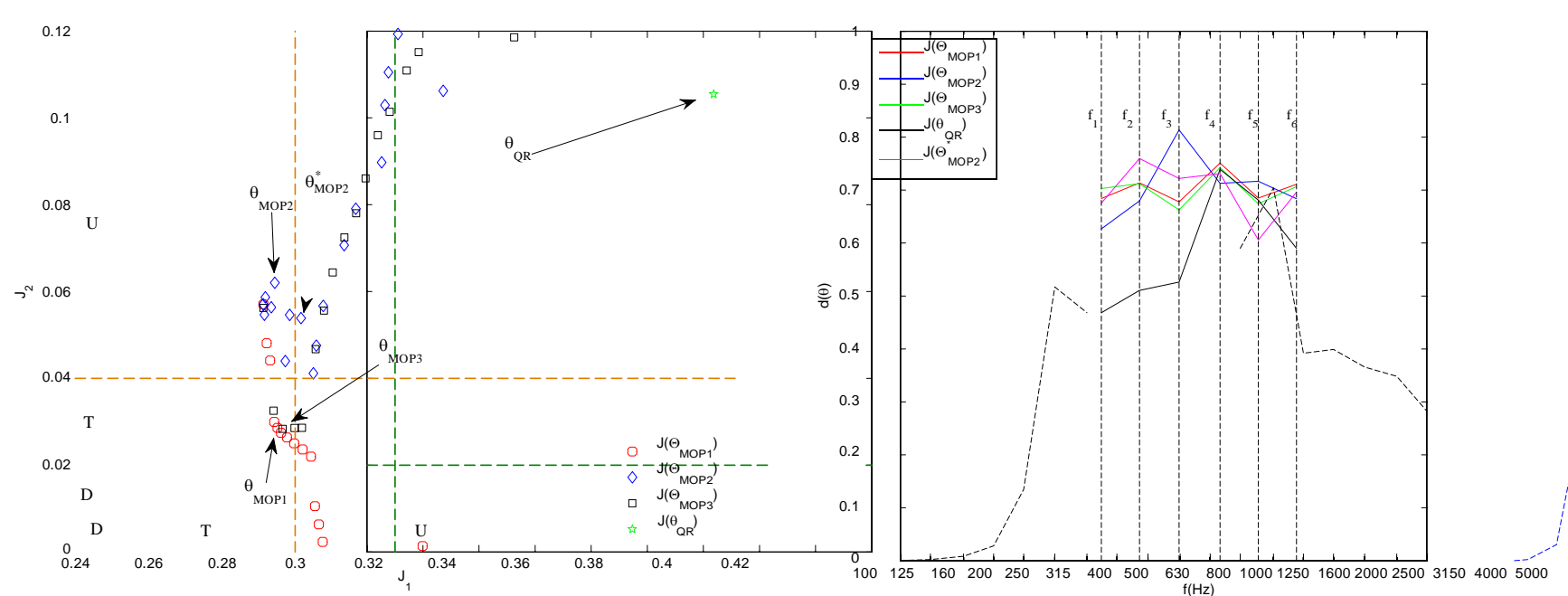

Fig. 15 Performance comparative for MOP1.

Fig. 18 Frequency response comparative for $\boldsymbol{\theta}_{M O P_{1}}$, $\boldsymbol{\theta}_{M O P_{2}}, \boldsymbol{\theta}_{M O P_{3}}, \boldsymbol{\theta}_{Q R}$ and $\boldsymbol{\theta}^{*}{ }_{M O P_{2}}$. Solid lines: Optimization range. Dashed lines: Out of the optimization range. No-

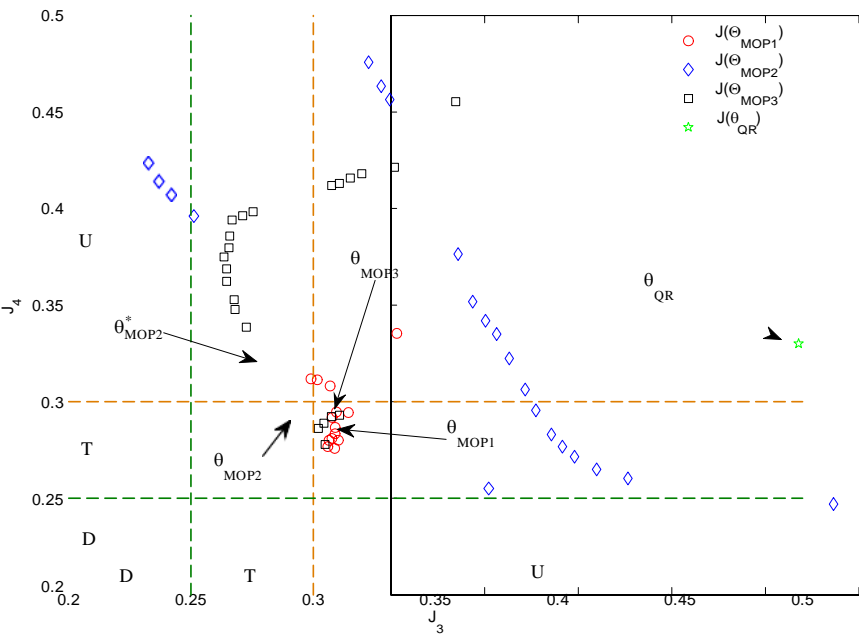
tice that frequencies over $1332 \mathrm{~Hz}$ are over the limit of validity of the Fraunhofer theory.

Table 7 Performance of $\boldsymbol{\theta}_{M O P_{1}}, \boldsymbol{\theta}_{M O P_{2}}, \boldsymbol{\theta}_{M O P_{3}}$ and $\boldsymbol{\theta}_{Q R}$ GPP-rank included.

\begin{tabular}{|l|c|c|c|c|c|}
\hline$\theta$ & $J_{1}, \mathrm{GPP}$ & $J_{2}, \mathrm{GPP}$ & $J_{3}, \mathrm{GPP}$ & $J_{4}, \mathrm{GPP}$ & $J_{5, \mathrm{GPP}}$ \\
\hline$\theta_{M P_{1} 1}$ & $0.296, \mathrm{~T}$ & $0.027, \mathrm{~T}$ & $0.308, \mathrm{U}$ & $0.284, \mathrm{~T}$ & $0.316, \mathrm{U}$ \\
$\theta_{M P_{2}}$ & $0.294, \mathrm{~T}$ & $0.062, \mathrm{U}$ & $0.293, \mathrm{~T}$ & $0.295, \mathrm{~T}$ & $0.373, \mathrm{U}$ \\
$\theta_{M P_{3}}$ & $0.299, \mathrm{~T}$ & $0.028, \mathrm{~T}$ & $0.307, \mathrm{U}$ & $0.292, \mathrm{~T}$ & $0.297, \mathrm{~T}$ \\
$\theta_{Q P}$ & $0.423, \mathrm{U}$ & $0.105, \mathrm{U}$ & $0.504, \mathrm{U}$ & $0.342, \mathrm{U}$ & $0.525, \mathrm{U}$ \\
\hline$\theta_{M P_{2}}^{*}$ & $0.301, \mathrm{U}$ & $0.053, \mathrm{U}$ & $0.280, \mathrm{~T}$ & $0.322, \mathrm{U}$ & $0.324, \mathrm{U}$ \\
\hline
\end{tabular}

Fig. 16 Performance comparative for MOP2.

the case of $\boldsymbol{\theta}_{\text {MOP } 2}$ that belongs to red subset represented in Fig.9.b. However in $\boldsymbol{\Theta}_{M O P 2}^{*}$ there is a solution with a similar structure to $\boldsymbol{\theta}_{M O P} 1$ and $\boldsymbol{\theta}_{M O P} 3$ (in subset represented in Fig. 9.c) and its performance approaches to $\boldsymbol{\theta}_{\text {MOP } 2}$ one (see Table 7). This solution is:

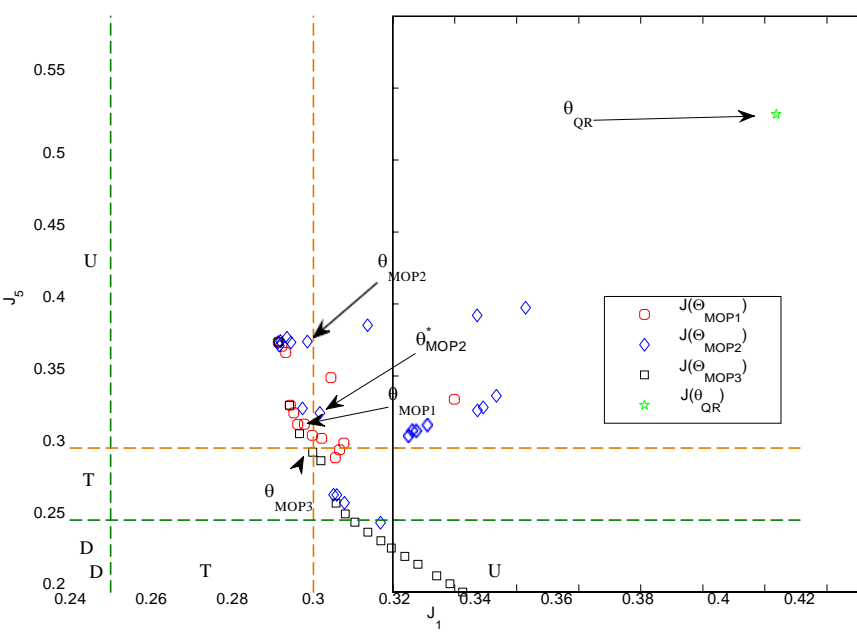

Fig. 17 Performance comparative for MOP3.

AMOP2 $=[100,0,182,278,96,298,216] \mathrm{mm}$

It seems that the structure of the solutions $\theta_{\text {MOP 2 }}^{*}$, $\theta_{M O P} 1$ and $\theta_{M O P} 3$ is a balanced solution for all the studied context, then a reasonable selection has to be done with this kind of structure. Among them we select $\theta_{\text {MOP } 3}$ (also $\boldsymbol{\theta}_{\text {MOP } 1 \text { could be a good alternative) since }}$ presents tolerable performance for $J_{1}, J_{2}, J_{3}$ and $J_{5}$ and it is only undesirable for $J_{4}$.

Finally, we would like to check that the use of the Fraunhofer theory is appropriate. As commented above, this theory is the simplest and the less accurate one. For comparison, we have obtained the reflection directivity sonogram using the Fraunhofer theory and FDTD [15]. Fig. 4.4 shows the diagram where the reflected acoustic pressure is represented as a function of both the angle and the frequency for $\theta_{M O P}$, calculated using FDTD and the Fraunhofer theory. One can see that, 


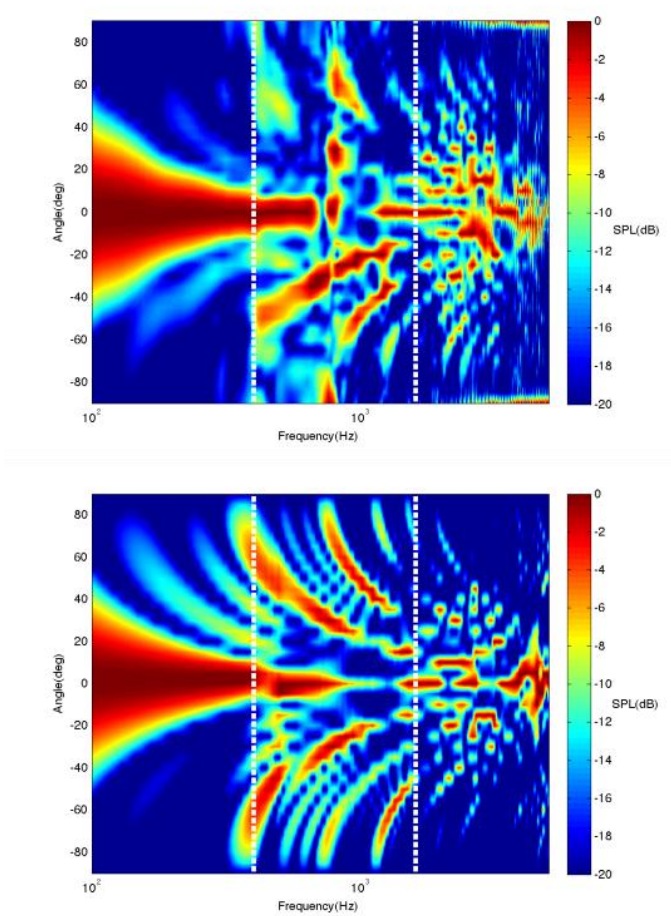

Fig. 19 Diagrams showing the reflected acoustic pressure field (Sound Pressure Level - SPL) of the $\theta_{M O P} 3$ individual as a function of the angle and the frequency calculated using both FDTD (above) and Fraunhofer theory (below). Vertical white lines delimit the frequency range in which diffusers have been optimized in this work.

although the results are not equal, they follow the same trend, supporting the use of the Fraunhofer theory in this work.

\section{Conclusions}

Along this paper a multiobjective optimization approach has been presented to design 1-D seven wells Schroeder sound diffuser, although this process could be applied to any kind of these diffusers. As far as we know only single-objective has been traditionally used for diffusers design. The multiobjective approach gives the designer the possibility to consider several properties simultaneously. When several objectives have to be optimized some of them are probably in conflict. That means there exists a set of possible solutions where the designers have to choose one according to their preferences. This kind of design requires a higher implication of the designer in the selection process. Multiobjective tools give the possibility to explore different sets of solutions and help to show the trade-off between them. Then the final solution is obtained through a process that gives more confidence or reliability to the designer.
The presented work uses an " a posteriori” MO approach where first the Pareto front is characterized and after a decision making process is performed based on a set of preferences. This procedure avoids to merge objectives in a single aggregated objective (the traditional " a priori" MO approach) and consequently avoids the problems associated with the construction of these kind of aggregated functions. Additionally" a posteriori" MO approach helps the designer to better understand the limitation of the design because it shows the complete trade-off among objectives.

As a particular improvement in the diffuser design procedure, we can find different diffusers that are equivalent. This fact produces multimodal optimization problems that is very important to avoid. For this purpose a specific codification of the diffusers is presented, forcing that one well is set to zero, avoiding flipped diffusers and considering the precision of manufacturing process. With this in mind, the number of possible diffusers with the codification proposed has been calculated and shows an important reduction of the search space. This reduction simplifies the optimization problem and increases the possibility to find a good approximation of the Pareto set. The ev-MOGA algorithm has been adequately modified to incorporate the codification presented in this paper.

Three bi-objective problems (MOP1, MOP2 and MOP3) have been set corresponding to different designer point of view. All the scenarios are based on the optimization of the normalized coefficient diffusion, but different points of view have been introduced changing the target ranges of frequencies.

The Pareto diffusers of MOP1, MOP2 and MOP3 and their Pareto fronts have been analysed. We would highlight that similar diffusers can produce quite different performance showing an important sensitivity. Due to the value of variation of the depth of the wells has been fixed in $2 \mathrm{~mm}$, the robustness analysis done (variations of plus/minus one step around the values of the Pareto front) shows in some cases an important loss of performances.

For some of the MOPs, the Pareto fronts obtained are disjoint and non convex justifying the use of a global multiobjective optimizer (for instance ev-MOGA). Additionally some of the selected solutions based on preferences (i.e. $\theta_{M O P}$ 1) were in non convex areas of the Pareto front. These solutions would be very difficult to localize with some traditional MO techniques.

The final diffuser selected $\left(\theta_{M O P}\right)$ is optimal in the Pareto sense and present a good trade-off among the objectives proposed. Finally we have obtained robust solutions since the acoustic performance of the proposed 
devices is hardly affected if little mistakes are produced in the manufacturing process of the diffuser.

\section{References}

1.Coello C, Veldhuizen D, Lamont G. Evolutionary algorithms for solving multi- objective problems. Kluwer Academic Publishers (2002).

2.Cox T.J. Predicting the scattering from reflectors and diffusers using $2 \mathrm{~d}$ boundary elements methods. J. Acoust.Soc. Am. 96 (1994).

3.Cox TJ. The optimization of profiled diffusers, J. Acoust. Soc. Am., 97, 2928-2936 (1995).

4.Cox TJ, D'Antonio P, Acoustic absorbers and diffusers. Theory, design and application. Second Edition, 290, Taylor \& Francis, London and New York(2009).

5.Herrero JM. Non-linear Robust identification using evolutionary algorithms, Ph.D. Thesis, Polytechnic University of Valencia, 2006

6.Herrero JM, et. al. Optimization of sonic crystal attenuation properties by ev-MOGA multiobjective evolutionary algorithm. Structural Multidisciplinary Optimization, 39(2), 203-215 (2009).

7.Herrero JM, Reynoso-Meza G, Martínez M, Blasco X, Sanchis J. A Smart-Distributed Pareto Front Using the evMOGA Evolutionary Algorithm. Int. J. Artif. Intell. Tools, 23(2) (2014).

8.Hughes RJ, Angus JAS, Cox TJ, Umnova O, Gehring GA, Pogson M, Whittaker DM, Volumetric diffusers: Pseudorandom cylinder arrays on a periodic lattice, J. Acoust. Soc. Am., 128(5), 2847-2856(2010).

9.ISO 17497-2:2012. Acoustics: Sound-scattering properties of surfaces. Part 2: Measurement of the directional diffusion coefficient in a free field.

10.Laumanns M, Thiele L, Deb K, Zitzler E. Combining convergence and diversity in evolutionary multi-objective optimization. Evolutionary computation, 10(3), 263-282 (2002).

11.Mattson CA, Muller A, Messac A.Smart Pareto Filter: Obtaining a minimal representation of multiobjective design space. Engineering Optimization, 36(6), (2004).

12.Mattson CA, Messac A. Pareto Frontier Based Concept Selection Under Uncertainty, with Visualization. Optimization and Engineering 6(1), 85-115(2005).

13. Messac A. Physical Programming: Effective Optimization for Computational Design. AIAA Journal, 34(1), 149158 (1996).

14. Miettinen KM. Nonlinear multiobjective optimization. Kluwer Academic Publishers, (1998).

15.Redondo J, Pico R, Roig B, Avis M. R. Time domain simulation of sound diffusers using finite-difference schemes, Acta Acustica uw Acustica, 93(4), (2007).

16.Sanchis J, Martínez M.A, Blasco X, Reynoso-Meza G. Modelling preferences in multiobjective engineering design. Engineering Applications of Artificial Intelligence 23, 12551264 (2010).

17.Schroeder MR, Diffuse sound reflection by maximumlength sequences, J. Acoust. Soc. Am., 57(1), 49-150 (1975).

18. Schroeder MR, Binaural dissimilarity and optimum ceilings for concert halls: more lateral sound diffusion, J. Acoust. Soc. Am., 65, 958-963 (1979).

19.Schroeder MR, Towards better acoustics for concert halls, Physics Today 33(10), 24-30 (1980).

20.Tusar T, Filipic B. Visualization of Pareto Front Approximations in Evolutionary Multiobjective Optimization: A Critical Review and the Prosection Method. IEEE Transaction on Evolutionary 19(2) (2014).
21.Zitzler E. Evolutionary algorithms for multiobjective optimization: Methods and applications. PhD thesis, Swiss Federal Institute of Technology Zurich (1999). 